\title{
Fatty acid oxidation disorders
}

\author{
J. Lawrence Merritt II ${ }^{1,2}$, Marie Norris ${ }^{2}$, Shibani Kanungo ${ }^{3}$
}

${ }^{1}$ Department of Pediatrics, University of Washington, Seattle, WA, USA; ${ }^{2}$ Biochemial Genetics, Seattle Children's Hospital, Seattle, WA, USA; ${ }^{3}$ Department of Pediatrics and Adolescent Medicine, Western Michigan University Homer Stryker MD School of Medicine, Kalamazoo, MI, USA Contributions: (I) Conception and design: All authors; (II) Administrative support: JL Merritt 2nd, S Kanungo; (III) Provision of study materials or patients: None; (IV) Collection and assembly of data: All authors; (V) Data analysis and interpretation: All authors; (VI) Manuscript writing: All authors; (VII) Final approval of manuscript: All authors.

Correspondence to: J. Lawrence Merritt II, MD. Associate Professor, Biochemical Genetics, Division of Genetic Medicine, Seattle Children's Hospital, Seattle, WA, USA; Department of Pediatrics, University of Washington, Seattle, WA, USA. Email: lawrence.merritt@seattlechildrens.org.

\begin{abstract}
Fatty acid oxidation disorders (FAODs) are inborn errors of metabolism due to disruption of either mitochondrial $\beta$-oxidation or the fatty acid transport using the carnitine transport pathway. The presentation of a FAOD will depend upon the specific disorder, but common elements may be seen, and ultimately require a similar treatment. Initial presentations of the FAODs in the neonatal period with severe symptoms include cardiomyopathy, while during infancy and childhood liver dysfunction and hypoketotic hypoglycemia are common. Episodic rhabdomyolysis is frequently the initial presentation during or after adolescence; although, these symptoms may develop at any age for most of the FAODs The treatment of all FAOD's include avoidance of fasting, aggressive treatment during illness, and supplementation of carnitine, if necessary. The long-chain FAODs differ by requiring a fat-restricted diet and supplementation of medium chain triglyceride oil and often docosahexaenoic acid (DHA) — an essential fatty acid, crucial for brain, visual, and immune functions and prevention of fat soluble vitamin deficiencies. The FAOD are a group of autosomal recessive disorders associated with significant morbidity and mortality, but early diagnosis on newborn screening (NBS) and early initiation of treatment are improving outcomes. There is a need for clinical studies including randomized, controlled, therapeutic trials to continue to evaluate current understanding and to implement future therapies.
\end{abstract}

Keywords: Fatty acid oxidation disorders (FAODs); mitochondrial $\beta$-oxidation; hypoketotic hypoglycemia; carnitine; trifunctional protein

Submitted Oct 02, 2018. Accepted for publication Oct 17, 2018.

doi: 10.21037/atm.2018.10.57

View this article at: http://dx.doi.org/10.21037/atm.2018.10.57

\section{Introduction}

When fat is needed as an energy source during periods of decreased intake, prolonged fasting, or increased energy demands due to illness, fatty acids are released from storage in the adipose tissue and undergo mitochondrial $\beta$-oxidation (1). Fatty acid $\beta$-oxidation is a major source of energy in the mitochondria; ultimately generating the reducing agents flavin adenine dinucleotide $\left(\mathrm{FADH}_{2}\right)$ and nicotinamide adenine dinucleotide $\left(\mathrm{NADH}^{+}\right)$to serve as electron donors to the respiratory chain for oxidative phosphorylation and ATP generation (Figure 1).
Fatty acid oxidation disorders (FAODs) lead to deficient energy production and produce three variable clinical presentations in all ages. Typically the most severe lifethreatening presentations may rapidly occur within a few hours of fasting in infants, but adults may require up to 48 hours of fasting. The neonatal-onset type, where newborns will develop a profound cardiomyopathy, hypoketotic hypoglycemia, and liver dysfunction within the first few days or weeks of life, is often fatal;. The infantileonset type will present in infancy or childhood with intermittent episodes of lethargy and vomiting associated with intercurrent illnesses and lead to hepatic dysfunction, 


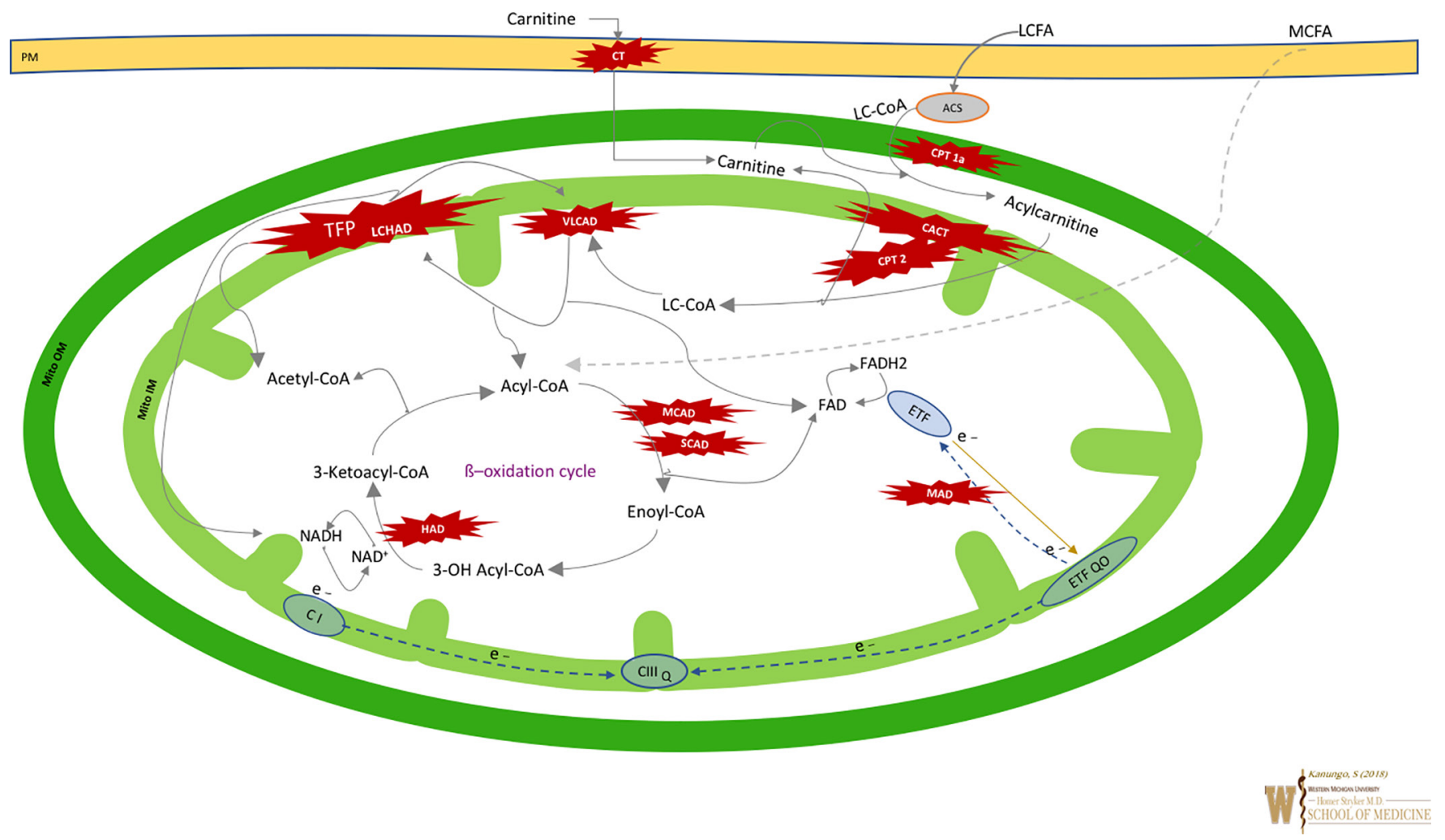

Figure 1 Overview of Mitochondrial Fatty Acid Oxidation Metabolism. Dark Green line, mitochondrial outer membrane (Mito OM); light green line, mitochondrial inner membrane (Mito IM); gold line, plasma membrane (PM). FAOD are shown in the red star shapes with associated enzyme defect. AC, Acyl-CoA; ACS, acyl-CoA synthase; CoA, coenzyme A; FA, fatty acids; MC, medium chain; LC, Long Chain; TFP, tri-functional protein complex; C I, complex I of ETC; CT, carnitine transporter defect; CPT1a, Carnitine palmitoyltransferase I; CPT2, carnitine palmitoyltransferase II; CACT, carnitine acylcarnitine translocase; LCHAD, long-chain 3-hydroxy acyl-CoA dehydrogenase; VLCAD, very long chain acyl-CoA dehydrogenase; MCAD, medium-chain acyl-CoA dehydrogenase; SCAD, short-chain acyl-CoA dehydrogenase; MAD, multiple acyl-CoA dehydrogenases; HAD, 3-hydroxyacyl-CoA dehydrogenase.

hypoketotic hypoglycemia, and encephalopathy or sudden death. The later- (or adolescent or adult-) onset myopathic type presents with episodes of muscle weakness, myalgias, rhabdomyolysis, and risk of renal damage.

\section{Genetics and epidemiology}

There is an estimated collective incidence of one in 5,00010,000 births, although the individual prevalence varies significantly (Table 1) (9). FAODs are all inherited in an autosomal recessive manner. Patients will have inherited two copies of a FAOD gene containing a DNA sequence mutation or variant. Recurrence risk for each pregnancy will then have a 1 in 4 chance of a child being affected; a 1 in 2 chance of being an unaffected carrier; and a 1 in 4 chance of being unaffected and not a carrier. Carriers are asymptomatic, though mild biochemical abnormalities may be present and detected on newborn screening (NBS).

\section{Initial presentations, diagnosis, and NBS}

Most FAODs are able to be detected through NBS by tandem mass spectrometry although there are profound differences between countries. For example, medium-chain acyl-CoA dehydrogenase deficiency (MCADD) deficiency is the only FAOD approved for NBS in the United Kingdom (10). Individuals with positive NBS are seen at metabolic centers for further testing before confirmation of a diagnosis. A plasma acylcarnitine profile and total and free carnitine levels are necessary for diagnosis following an abnormal NBS. The FAOD can all be recognized by their specific acylcarnitine profiles and free carnitine 
Table 1 Genetics of fatty acid oxidation disorders

\begin{tabular}{llll}
\hline Disorder & Gene & Prevalence & Common sequence variants \\
\hline MCADD & ACADM & $\begin{array}{l}1: 20,000 \text { in Northern } \\
\text { European Caucasian }\end{array}$ & $\begin{array}{l}\text { c.985A }>\mathrm{G} \text { (p.K304E) in approximately } 70 \% \text { of mutant alleles in } \\
\text { affected patients of Northern European }\end{array}$ \\
VLCADD & ACADVL & $1: 42,500$ to $1: 120,000$ & mild or benign DNA variant p.V283A (2) \\
LCHADD & HADHA & $1: 110,000$ to $1: 150,000$ & c.1528-G>C (p.E510Q) \\
TFPD & HADHA, HADHB & Rare & - \\
CPT1D & CPT1A & $1: 500,000$ & Milder phenotype c.1436C $>$ T (p.P479L) in Inuit, Alaskan native, \\
CACTD & SLC25A20 & Rare & - \\
CPT2D & CPT2 & Rare & p.S113L in 60\% later-onset myopathic presentation (6) \\
CTD & SLC22A5 & $1: 20,000$ to 1:120,000 & - \\
SCADD & ACADS & $1: 35,000$ to 1:50,000 & c.511C>T and c.625G $>$ A in 14\% of normal population (7,8) \\
MADD & ETFA, ETFB, ETFDH & Rare & - \\
HADD & HADH & Rare & - \\
\hline
\end{tabular}

MCADD, medium-chain acyl-CoA dehydrogenase deficiency; VLCADD, very long-chain acyl-CoA dehydrogenase deficiency; LCHADD, long-chain 3-hydroxy acyl-CoA dehydrogenase deficiency; TFPD, trifunctional protein deficiency; CPT1D, carnitine palmitoyltransferase type 1 deficiency; CACTD, carnitine-acylcarnitine translocase deficiency; CPT2D, carnitine palmitoyltransferase type 2 deficiency; CTD, carnitine transporter deficiency (CTD); SCADD, short-chain acyl-CoA dehydrogenase deficiency; MADD, Multiple acyl-CoA dehydrogenase deficiency; HADD, 3-hydroxyacyl-CoA dehydrogenase deficiency

levels (Table 2). Testing of urine organic acids or urine acylglycines may be helpful for some FAOD and reveal a diagnostic pattern of dicarboxylic acids or acylglycines. DNA testing is considered standard for confirmation and can be helpful in genotype/phenotype correlations. DNA sequencing may reveal variants of uncertain significance, so further investigation of enzyme activity through fibroblast, leukocyte, or liver enzyme testing or by oxidized labeled fatty acids in fibroblasts (e.g., FAO probe) may provide additional information of functional significance.

The presentation of a FAOD will depend upon the specific disorder, but common elements may be seen, and ultimately require a similar treatment (discussed below). During an acute presentation, a patient may have hypoglycemia with hypoketosis. Serum or urine ketones are often not present in FAOD but are not specific. Elevations of transaminases, ammonia, and creatine kinase may be present. A metabolic acidosis may be found due to dehydration secondary to the underlying illness and with hypoglycemia. Cardiac evaluation is essential the in acute presentation by chest radiography, electrocardiogram, or echocardiogram for the long-chain fatty acid oxidation disorders (LCFAOD).

False positive NBS are relatively common for FAOD, partially due to a higher detection rate of carriers or apparently milder forms of FAOD. These infants have presented a unique clinical challenge as they may have persistent but lower concentrations of their respective acylcarnitine species and remain asymptomatic when compared to the more severe forms of FAOD. These infants may have a risk for late-onset disease and there is a significant need for additional research and long-term follow-up studies (11).

\section{Fatty acid oxidation disorders (FAOD)}

\section{Medium-chain acyl-CoA dehydrogenase deficiency (MCADD)}

MCADD is the most common FAOD (12). While historically MCADD had a mortality rate of at least $20 \%$ prior to early diagnosis through NBS (13), with early implementation of treatment following NBS mortality and morbidity have improved to as low as $5 \%(14,15)$. MCADD may present in infancy and in toddlers associated with infection and resulting in vomiting and poor oral intake, progressing to dehydration, lethargy, and hypoketotic hypoglycemia. Liver dysfunction can present clinically similar to Reye syndrome. Progression of disease will lead to death from hyperammonemia and brain edema following 


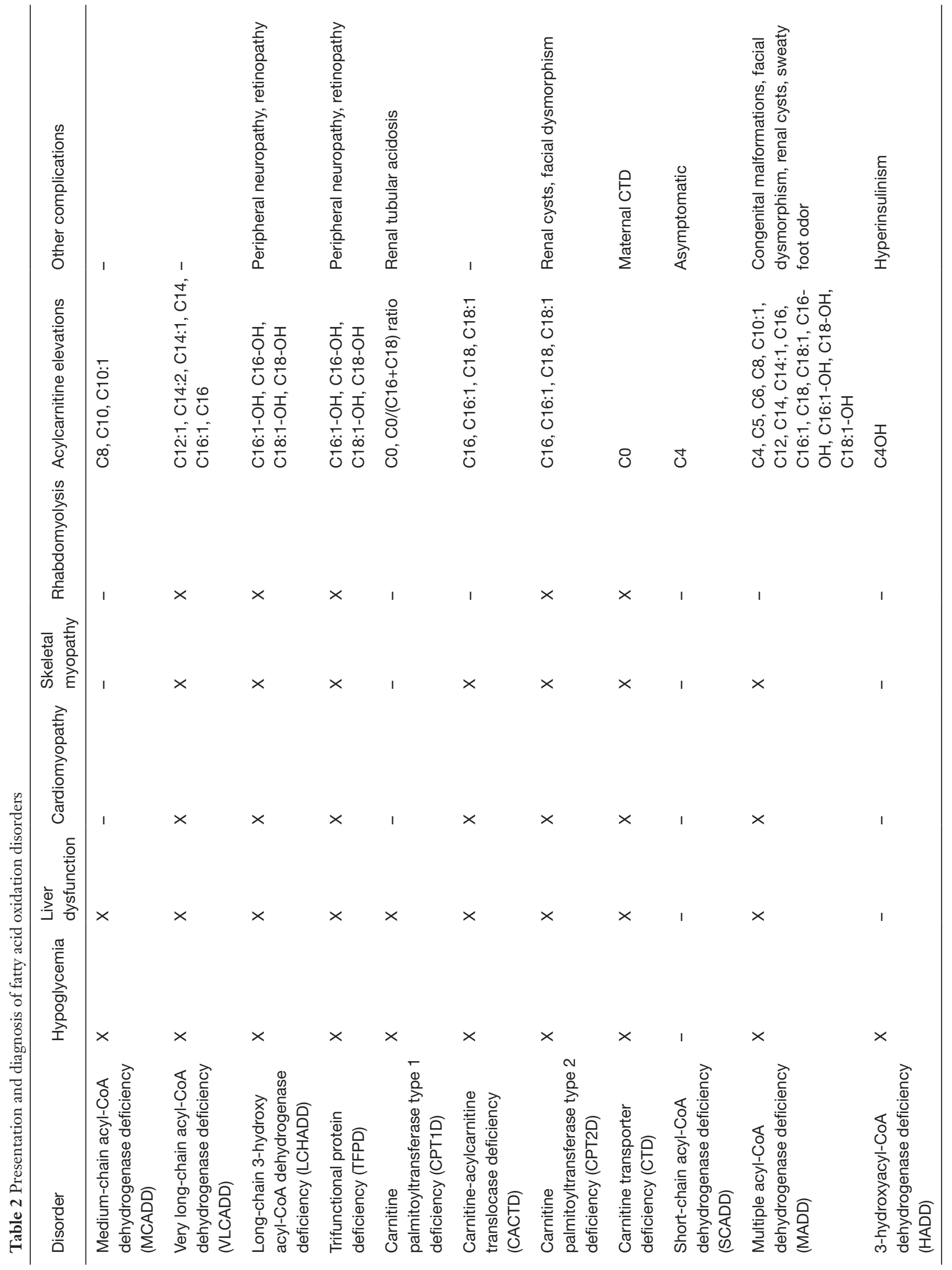


lack of treatment from unrecognized disease, including sudden lethal presentations in all age groups (16).

Acylcarnitine abnormalities from NBS should be confirmed in plasma samples (Table 2). Urine acylglycine testing shows elevations of propionylglycine, suberylglycine and hexanoylglycine. DNA sequencing of $A C A D M$ is readily available and homozygosity for the c. $985 \mathrm{~A}>\mathrm{G}$ mutation is often associated with the most severe phenotype, including sudden death in the first 72 hours of life prior to receiving the results of NBS (17).

\section{Very long-chain acyl-CoA debydrogenase deficiency (VLCADD)}

The prevalence of VLCADD now has increased following NBS (18-21). Complete deficiency may present with severe cardiomyopathy and death in the first few days of life. Partial deficiency may only have recurrent hypoketotic hypoglycemia or presentation in adolescence or adulthood with myopathy and/or rhabdomyolysis (2,22-25).

Diagnosis following lethal cardiomyopathy may only be detected post-mortem or on NBS results. NBS will detect VLCADD, although many countries do not screen for VLCADD due high false positive rates and uncertainty with managing and treating milder disease variants (2). Plasma acylcarnitines may confirm the diagnosis, although profiles may be normal when the patient isn't in acute metabolic stress, so sequencing of $A C A D V L$ is recommended for confirmation. Urine organic acid analysis may demonstrate longer-chain dicarboxylic aciduria during illness and metabolic stress. These patients with single heterozygous or novel variants may have persistent acylcarnitine elevations and leukocyte enzyme assay and/or FAO probe analysis may help determine the need for treatment (26).

\section{Long-chain 3-hydroxy acyl-CoA debydrogenase deficiency (LCHADD) and trifunctional protein deficiency (TFPD)}

The mitochondrial trifunctional protein heterooctomer includes the four alpha and four beta subunits encoded by the $H A D H A$ and $H A D H B$ genes, respectively. The mitochondrial trifunctional protein complex has three enzymatic activities: long-chain enoyl-CoA hydratase, longchain 3-hydroxy acyl-CoA dehydrogenase, and 3-ketoacylCoA thiolase. TFPD is due to deficiency of all three enzymes resulting from mutations in either $H A D H A$ or $H A D H B$. LCHADD occurs from mutations in $H A D H B$ resulting in deficiency of the dehydrogenase subunit.
Mothers who are heterozygous carriers for LCHADD have been reported to have a risk of developing acute fatty liver of pregnancy during pregnancy and hemolysis, elevated liver enzymes, low platelets (HELLP) syndrome when carrying an affected fetus-and so their children should have appropriate screening at birth (10).

The most severe presentation of neonatal LCHADD and TFPD is a rapidly progressive cardiomyopathy $(27,28)$. Infantile presentations during times of acute illness can include recurrent hypoketotic hypoglycemia and liver dysfunction (e.g., a Reye-like syndrome), cholestasis, cardiomyopathy, myopathy, and rhabdomyolysis. Longterm development of skeletal myopathy (65\%), slowly progressing peripheral neuropathy (21\%), and pigmentary retinopathy (43\%) require clinical monitoring but can be difficult to treat (28). Peripheral neuropathy may be more severe and present at earlier ages in TFPD. Severe liver disease may progress to necrosis and steatosis. Episodes of recurrent rhabdomyolysis are common in older children, adolescents, and adults. Improved growth a development are now being seen due to NBS and early institution of therapy in LCHADD although prevention of all morbidity and mortality are incomplete, especially in TFPD patients $(27,29)$. The survival rate for TFPD is worse than LCHADD (27-30).

Diagnostic elevations are seen on plasma acylcarnitine analysis (Table 2). Longer-chain 3-hydroxydicarboxylic acids may be present in urine organic acid analysis. A leukocyte or fibroblast enzyme assay is available although these assays have significant overlap between heterozygous carriers and those with two mutations; however, DNA sequencing demonstrating mutations in $H A D H A$ or $H A D H B$ is typically adequate.

\section{Carnitine transport disorders}

Long-chain fatty acids require carnitine for transport across the mitochondrial inner membrane. This three step process involves covalently linking carnitine to the longchain fatty acyl-CoA via a transferase (CPT1), movement across the inner mitochondrial membrane by a sodiumdependent carnitine transporter, and then removal of the long-chain fatty acyl-CoA from carnitine by a second transferase (CPT2).

\section{Carnitine palmitoyltransferase type 1 deficiency (CPT1D)}

The three different isoforms of the CPT1 enzyme are 
encoded by different genes, but only CPT1A deficiency, expressed in the liver and kidney, has been reported. Early childhood presentations of CPT1D include hypoketotic hypoglycemia, liver dysfunction, and rapid progression to liver failure. Neonatal hypoglycemia is rare and adult-onset skeletal myopathy is infrequent (31). Renal tubular acidosis has been reported with epidoses of acute decompensation (32).

NBS for CPT1D is available in most states and laboratory findings include elevated free plasma carnitine levels with decreased levels of long-chain acylcarnitines. An abnormal ratio of $\mathrm{C} 0 /(\mathrm{C} 16+\mathrm{C} 18)$ improves specificity in NBS. DNA sequencing of the $C P T 1 A$ gene or fibroblast enzyme activity is available. A milder phenotype of CPT1D is seen at a higher prevalence in the Arctic populations may be associated with higher infant mortality and impaired fasting intolerance $(3-5,33)$.

\section{Carnitine-acylcarnitine translocase deficiency (CACTD)}

The most severe presentation of CACTD is with severe neonatal cardiomyopathy, ventricular dysrhythmias, hypoglycemia, hyperammonemia, and sudden death $(30,34)$. Symptoms in older patients include vomiting, hypoglycemia, mild chronic hyperammonemia, severe skeletal myopathy, and mild hypertrophic cardiomyopathy. Many patients may still have significant developmental delay, seizures, and other complications despite early diagnosis through NBS and implementation of treatment (30,35-37). Other patients may have a less severe disease associated with higher enzyme activity, although disease severity does not always correlate with disease severity $(38,39)$.

Findings from NBS and diagnosis from plasma acylcarnitines should be confirmed with DNA sequencing of SLC25A20 because the acylcarnitine profiles on NBS or plasma are identical to carnitine palmitoyltransferase type 2 deficiency (CPT2D). Even with NBS, prognosis is poor in most patients and mortality remains high $(30,37)$. Surviving patients may develop profound developmental delay and seizures even with treatment $(35,36)$.

\section{Carnitine palmitoyltransferase type 2 deficiency (CPT2D)}

CPT2D is most commonly recognized by its later-onset myopathic presentation in adolescence or adulthood due to exercise intolerance and episodes of rhabdomyolysis with associated risk of renal failure (40). Additionally, there is a rare, severe, neonatal form presentation with where infants will have multiple congenital anomalies (dysmorphic facies, renal dysgenesis, neuronal migration malformations), hypotonia, cardiomyopathy, arrhythmias, seizures, but may result in death as early as the first few days of life (40). Acylcarnitines in CPT2D and CACTD are identical, so confirmatory sequencing of the gene, $C P T 2$, is necessary (6).

\section{Carnitine transporter deficiency (CTD)}

CTD is also known as carnitine uptake defect or primary carnitine deficiency (41). CTD presents with hypoketotic hypoglycemia, hyperammonemia, liver dysfunction, cardiomyopathy, and skeletal hypotonia. Neonatal presentations are unusual, while children may present with cardiomyopathy. On NBS affected infants have profoundly low free carnitine levels and diagnosis requires plasma total and free carnitine levels in both the neonate and mother. False positive NBS for low free carnitine levels have been reported due to neonatal nutritional deficiency or previously undetected low maternal plasma carnitine levels resulting from either unrecognized maternal CTD or a low-protein diet (e.g., vegan diet). Women with maternal CTD may be either asymptomatic or have unrecognized clinical symptoms and may or may not clinically benefit from carnitine supplementation. DNA sequencing of the SLC22A5 gene is adequate confirming the diagnosis or fibroblast carnitine uptake analysis is available (41). Patients with CTD only require carnitine supplementation as their treatment and do not require dietary restrictions (41).

\section{Other FAOD}

\section{Short-chain acyl-CoA dehydrogenase deficiency (SCADD)}

Previously, decreased SCAD enzyme activity was considered to be associated with symptoms ranging from poor feeding, failure to thrive, hypotonia, and seizures. Subsequently, compound heterozygosity or homozygosity for one of two common polymorphisms (Table 1) were found to result in a reduced enzyme activity and the biochemical abnormalities of SCADD but not thought to be of any physiologic consequence (42).

SCADD is diagnosed through elevations of C4acylcarnitine on NBS or on plasma acylcarnitine profiles. Urine organic acids show elevations of ethylmalonic acid and butyrylglycine. NBS will detect infants with mild, moderate and severely decreased enzyme function. Currently all 
infants with SCADD detected on NBS have been reported to remain asymptomatic, leading many to consider SCADD to be a benign biochemical phenotype rather than a clinically relevant inborn error of metabolism (7). The need for treatment of SCADD is uncertain, and the need for acute management does not appear necessary.

\section{Multiple acyl-CoA dehydrogenase deficiency (MADD)}

MADD (also known as glutaric acidemia type 2; GA2) is a complex inborn error of metabolism due to a combined defect multiple acyl-CoA dehydrogenases involving amino acid, fatty acid, and choline metabolism. The electron transfer flavoprotein (ETF) is made up of two subunits: ETFA and ETFB. Mutations in the genes, ETFA, ETFB, and ETFDH, disrupt the electron transfer from ETF to electron transfer flavoprotein dehydrogenase (ETFDH) and then ultimately to coenzyme Q10 in complex III of the mitochondrial electron transport chain. Riboflavin is a precursor of the FAD cofactor and riboflavin responsive mutations have been described, most frequently in ETFDH, with a milder, riboflavin-responsive phenotype (43). Riboflavin deficiency or other riboflavin metabolism disorders may have a similar acylcarnitine profile to MADD.

Three overlapping clinical presentations of MADD are recognized. Neonates may or may not have congenital anomalies (such as enlarged polycystic kidneys, rockerbottom feet, inferior abdominal musculature defects, hypospadias and chordee, cerebral cortical dysplasia, and gliosis). Dysmorphic features may include macrocephaly, large anterior fontanel, telecanthus, malformed ears, high forehead, and flat nasal bridge, similar to those in severe CPT2D (44). There is a significant risk of sudden progression of hypertrophic cardiomyopathy and sudden death despite early diagnosis on NBS with early initiation of treatment $(45,46)$. Later-onset MADD, which is more likely to be riboflavin responsive, does not have congenital malformations, but does have continued risk of acute intermittent episodes with vomiting, dehydration, hypoketotic hypoglycemia, and acidosis with hepatomegaly or myopathy.

NBS will detect MADD and plasma acylcarnitines and urine organic acids will confirm the diagnosis (Table 2). Metabolic abnormalities include lactic acidosis with an increased anion gap metabolic acidosis, hypoketotic hypoglycemia, and hyperammonemia. The "sweaty feet" odor of isovaleric acid may be present. Urine organic acid testing shows elevations of ethylmalonic acid, glutaric acid, 3-hydroxyisovaleric acid, lactic acid, medium- and long-chain dicarboxylic acids, and isovalerylglycine, isobutyrylglycine, and 2-methylbutyrylglycine. Renal tubular dysfunction results in generalized aminoaciduria. DNA sequencing of ETFA, ETFB, and ETFDH is helpful given the known riboflavin responsiveness and metabolism disorders. Enzyme analysis from liver biopsy or fibroblast fatty acid oxidation probe analysis is available, if necessary.

\section{3-bydroxyacyl-CoA debydrogenase deficiency (HADD)}

This disorder has previously been referred to as medium/ short-chain 3-hydroxyacyl-CoA dehydrogenase deficiency (M/SCHADD). Encoded by the HADH gene, this dehydrogenase catalyzes the $\mathrm{NAD}^{+}$dependent oxidation of 3-hydroxyacyl-CoA for C4-10 substrates $(47,48)$. HADD should be classified as a congenital hyperinsulinism syndrome as patients with proven $H A D H$ mutations may have recurrent hypoglycemia with a diazoxide-responsive hyperinsulinism (49-52). Hyperinsulinism and protein sensitivity results from a nonenzymatic function of the HAD protein inhibiting the activation of glutamate dehydrogenase (GDH) leading to gain of activity insulin sensitivity (53). Stressors, fasting, or dietary protein may provoke symptoms. HADD patients reported to date present within the first year of life. Late diagnosis can result in seizures and mental retardation due to recurrent hypoglycemia (51). During episodes of hypoglycemia and on NBS, mild hyperammonemia and elevations short- and medium-chain 3-hydroxyacylcarnitines may be found.

\section{Nutrition management of FAOD}

\section{Summary of common elements of treatment}

Nutrition management of all FAODs includes avoidance of fasting, aggressive treatment during illness, and supplementation of carnitine, if necessary (Table 3). These three treatment strategies are congruent among all FAODs but there are some differences in nutrition management for specific FAODs.

\section{Medium-chain acyl-CoA dehydrogenase deficiency (MCADD)}

Infants with MCADD require frequent feedings dependent on their age as discussed in the summary (Table 3). After infancy most toddlers, children, and adults require regular meals and snacks during the day and before bed to prevent 
Table 3 Summary of nutrition management for all FAODs

\begin{tabular}{|c|c|}
\hline Category & Common elements of nutrition management \\
\hline \multirow[t]{2}{*}{ Avoidance of fasting } & Infants need to be fed every 3 hours with no more than 4 hours of fasting age $0-4$ months; \\
\hline & An additional hour of fasting is allowed per month of age thereafter up to 8 hours until 12 months of age; \\
\hline \multirow{2}{*}{$\begin{array}{l}\text { Aggressive treatment during } \\
\text { increased metabolic stress }\end{array}$} & Providing oral or enteral carbohydrate-rich fluids every 3-4 hours with mild to moderate illness; \\
\hline & $\begin{array}{l}\text { Intravenous fluids with } 10 \% \text { dextrose at } 1.5 \text { times maintenance rate with appropriate electrolytes are } \\
\text { provided during times of illness, poor oral intake, or when fasting for surgery (28) }\end{array}$ \\
\hline \multirow[t]{2}{*}{ Supplementation of carnitine } & Carnitine supplementation is controversial; \\
\hline & $\begin{array}{l}\text { Carnitine supplementation may only be helpful if secondary carnitine deficiency is found, although some } \\
\text { mouse studies have suggested long-chain hydroxyl-acylcarnitines may induce arrhythmias (54) }\end{array}$ \\
\hline
\end{tabular}

hypoglycemia and fatigue (55). Children and adults with MCADD should eat a normal, healthy diet (30\% of energy from fat) that is appropriate for their age. Patients with MCADD do not need more food than the average person, but they need to eat healthy food at regular intervals.

Breastmilk or standard infant formulas are appropriate to meet nutrient needs during infancy with introduction of solids per standard infant feeding guidelines. It is important for patients with MCADD to avoid medium chain triglyceride (MCT) oil. Some specialty infant and pediatric formulas have higher amounts of MCT oil and should be avoided (Table 4). If infants are on one of these specialty formulas, they may have false positive NBS results indistinguishable from those of a newborn with MCADD (55).

Dietary sources of MCT are typically difficult to find, with the exception of manufactured MCT oil ${ }^{\circledR}$ and coconut oil. Patients with MCADD are educated to avoid excessive consumption of coconut oil, but are permitted to have foods that contain coconut as an ingredient.

During illness aggressive actions must be taken especially if the child is vomiting or not eating well. Families are provided with an emergency protocol/letter that can be carried with them at all times and are strongly advised to seek medical attention if the patient with MCADD is experiencing illness accompanied with the previously mentioned symptoms. During milder illnesses, if the child is able to drink fluids they need to drink carbohydrate-rich fluids such as Gatorade ${ }^{\circledR}$ or Pedialyte ${ }^{\circledR}$ mixed with Solcarb ${ }^{\circledR}$ (powdered carbohydrate) every 3-4 hours during the day and night to maintain blood sugar and hydration. If fluids are not tolerated and/or the symptoms progress that signs of dehydration or mental status changes are noted, the child needs to be brought to the emergency department with their emergency letter for intravenous fluids as mentioned in the nutrition management summary (55).

\section{Long-chain fatty acid oxidation disorders (LCFAOD)}

Treatment of LCFAODs is similar to MCADD regarding avoiding fasting, providing aggressive treatment during illness, and possible supplementation with carnitine, if deficient. LCFAODs differ by requiring a fat restricted diet, potentially a higher protein intake, and supplementation of MCT (as a substrate for $\beta$-oxidation) (1).

The primary goal of nutrition management of LCFAODs is to limit long chain fat as a substrate for energy production both by preventing $\beta$-oxidation and catabolism and by limiting the amount of dietary long chain fat while still providing adequate nutrients for normal growth and development (1). The extent to which fat is restricted is dependent on each patient's gene mutation and severity of their disease. LCFAOD patients with mild or moderate forms usually only experience intermittent symptoms at times of increased $\beta$-oxidation such as during muscle during exercise or while fasting (56). 
Table 4 MCT oil content in specialty infant and pediatric formulas

\begin{tabular}{|c|c|c|}
\hline Formula type & Formula name & $\% \mathrm{MCT}$ \\
\hline \multirow{6}{*}{$\begin{array}{l}\text { Premature infant } \\
\text { formulas }\end{array}$} & Enfamil human milk fortifier ${ }^{a}$ & 70 \\
\hline & Enfamil premature $^{a}$ & 40 \\
\hline & Enfacare $^{a}$ & 20 \\
\hline & Neosure $^{b}$ & 25 \\
\hline & Similac human milk fortifier ${ }^{\mathrm{b}}$ & 100 \\
\hline & Similac special care ${ }^{b}$ & 50 \\
\hline \multirow{4}{*}{$\begin{array}{l}\text { Semi-elemental/ } \\
\text { hypoallergenic infant } \\
\text { formulas }\end{array}$} & Alimentum $^{\mathrm{b}}$ & 50 \\
\hline & Pregestimil $^{\mathrm{a}}$ & 55 \\
\hline & Elecare $^{\mathrm{b}}$ & 33 \\
\hline & Neocate $^{c}$ & 33 \\
\hline \multirow{7}{*}{$\begin{array}{l}\text { MCT supplemented } \\
\text { metabolic formulas for } \\
\text { LCFAODs }\end{array}$} & Lipistart (infant) ${ }^{\mathrm{d}}$ & 90 \\
\hline & Monogen (infant) $^{c}$ & 80 \\
\hline & Portagen (infant) ${ }^{a}$ & 87 \\
\hline & Enfaport (infant) ${ }^{a}$ & 83 \\
\hline & MCT Procal ${ }^{d}$ (pediatric) & 97 \\
\hline & MCT oile (pediatric) & 100 \\
\hline & Liquigen $^{c}$ (pediatric) & 96 \\
\hline
\end{tabular}

${ }^{\mathrm{a}}$, Mead Johnson Nutrition Evansville, IN; ${ }^{\mathrm{b}}$, Abbott Nutrition, Lake Forest, IL; ${ }^{\text {, }}$ Nutricia North America, Rockville, MD; ${ }^{\text {, }}$, Vitaflo USA, Alexandria, VA; ${ }^{e}$, Nestle Nutrition, Florham Park, NJ.

LCFAOD nutrition management in infancy includes total fat intake from all sources (MCT and long chain fat) to provide $40-45 \%$ of energy (typical for a normal infant). In severe VLCADD, long chain fat is restricted to $10 \%$ and MCT to $30 \%$ of energy. For moderate or mild forms of VLCADD, this may be $20 \%$ of energy from long chain fat and $20 \%$ of energy from MCT (1). Symptomatic infants need to discontinue breastfeeding (due to the high fat content in breast milk) and start specialized MCTsupplemented formula specific to LCFAODs (Table 4) (57). These formulas are prescribed by the metabolic physician. Breastfeeding may be accompanied with MCT-supplemented formula in patients with milder or asymptomatic forms of VLCADD.

In childhood and adulthood the allowance of fat energy decreases to $30 \%$ of energy after 1 year of age. In severe forms the patient will still need to restrict long chain fat to $10 \%$ of energy and provide MCT as $20-30 \%$ of energy. Patients with mild or moderate forms require long chain fat restricted to $15-20 \%$ of energy and provide MCT as $15-$ $20 \%$ of energy (1). Some metabolic centers will alternatively dose MCT at $2-3 \mathrm{~g} / \mathrm{kg}$ in infancy and $1-1.5 \mathrm{~g} / \mathrm{kg}$ after 1 year of age (58). Since there is residual enzyme activity, there are ongoing studies to determine if mild or moderate forms even need a long chain fat restriction if they are anabolic. Age appropriate solid foods are introduced around 4-6 months of age (which is typical for any average infant). After a year of age more solid foods are introduced to meet their nutrition needs. As the child increases the amount of solid foods in their diet, their supply of long-chain fats comes from these sources. Patients are prescribed a daily limit in the number of grams of long chain fat allowed per day and are educated about how to count grams of fat in food.

After 1 year of age, MCT can be supplemented as an oil $\left(\right.$ MCT oil $^{\circledR}$ or Liquigen ${ }^{\circledR}$ ) or as a powder (MCT Procal ${ }^{\mathrm{TM}}$ ), which should be prescribed by the metabolic physician. Some patients mix MCT in nonfat milk and drink it at regular intervals throughout the day and some mix their MCT into their foods. MCT supplementation has been associated with a reversal of cardiomyopathy in CACTD and VLCADD although some VLCADD patients still had significant muscle weakness, muscle pain, or myoglobinuria $(28,35,59,60)$. Since MCT infant formulas are enriched with essential fatty acids, it is important to provide adequate essential fats when infant formula is discontinued. Essential fatty acid linoleic acid (C18:2n6) should compose $3 \%$ of energy intake and $\alpha$-linolenic acid (C18:3n3) should comprise $1 \%$ of energy intake (1).

Avoiding essential fatty acid deficiency is important, and the majority of long chain fat consumption should come from oils rich in essential fatty acids such instead of saturated long chain fatty acids (i.e., butter, fatty meats, etc.) (1). Supplementation with specific oils such as walnut or flaxseed oil may be necessary to meet essential fatty acid requirements (Table 5). Patients are at higher risk for becoming deficient in fat soluble vitamins and may require supplementation (62).

Linoleic acid and $\alpha$-linolenic acid are precursors for the endogenous synthesis of longer fatty acids such as eicosapentaenoic acid (EPA; C20:5n3) and docosahexaenoic acid (DHA; C22:6n3), which are crucial for brain, visual, and immune functions (62). It is often difficult to increase plasma EPA and DHA concentrations from dietary oils alone so patients require supplementation with preformed DHA (60 mg/day for infants $<20 \mathrm{~kg} ; 100 \mathrm{mg}$ /day for children $>20 \mathrm{~kg}$; and 100-200 mg/day for adults) to achieve 
Table 5 Sources of essential fatty acids (61)

\begin{tabular}{llllll}
\hline Source & Amount & $\begin{array}{l}\text { Long-chain } \\
\text { fat }(\mathrm{g})\end{array}$ & $\begin{array}{l}\text { Linoleic } \\
\text { acid }(\mathrm{mg})\end{array}$ & $\begin{array}{l}\text { Linolenic } \\
\text { acid }(\mathrm{mg})\end{array}$ & $\begin{array}{l}\text { Energy } \\
(\text { Kcal })\end{array}$ \\
\hline Flaxseed oil & $1 \mathrm{~mL}$ & 0.9 & 114 & 480 & 8 \\
Canola oil & $1 \mathrm{~mL}$ & 0.9 & 183 & 84 & 8 \\
Walnut oil & $1 \mathrm{~mL}$ & 0.9 & 476 & 94 & 8 \\
Safflower oil & $1 \mathrm{~mL}$ & 0.9 & 672 & 0 & 8 \\
Corn oil & $1 \mathrm{~mL}$ & 0.9 & 482 & 10 & 8 \\
Soy oil & $1 \mathrm{~mL}$ & 0.9 & 459 & 61 & 8 \\
\hline
\end{tabular}

normal levels (62). All patients with LCFAODs should be given DHA, as DHA supplementation may stabilize, but not prevent, the retinopathy seen in LCHADD (63).

With a fat restricted diet, patients with LCFAODs are potentially at risk for inadvertently lowering their protein intake unless they specifically consume low fat, high protein foods (e.g., nonfat dairy, lean meats). An investigation of a higher protein diet in LCHADD and TFPD patients showed a higher protein diet with less carbohydrates does not improve metabolic control, but may be beneficial for body composition and liver lipid content (62).

During exercise, the body utilizes glycogen from muscle to provide the majority of energy for the first 20-30 minutes. Prolonged exercise and higher intensity exercise is dependent on fatty acids. Patients with LCFAODs are at high risk of exercise-induced rhabdomyolysis. To prevent rhabdomyolysis and increase exercise tolerance, patients require additional MCT supplementation $(0.15-0.2 \mathrm{~g} / \mathrm{kg}$ weight) mixed with a glucose solution (e.g., Gatorade ${ }^{\circledR}$ ) prior to exercise and a 3:1 ratio of carbohydrate to protein snack after exercise (62).

Patients with LCFAODs are always at risk of metabolic decompensation especially during stress and any instance where energy intake is decreased (e.g., illness, fasting, vigorous exercise, skipping meals, vomiting, surgical procedures). Just like with MCADD, during illness patients need to drink carbohydrate-rich fluids every 3-4 hours along with their MCT. If oral intake is not tolerated, the illness lasts more than three days, and/or the patient is lethargic, the patient needs to be brought to the emergency department for intravenous fluids (Table 3) (58). MCT should be reinstituted as soon as the patient is able to tolerate oral/enteral intake. If parenteral nutrition is implemented, Intralipid ${ }^{\circledR}$ is contraindicated (1).

\section{MADD}

Nutrition management of MADD is complex and requires a low fat (20-25\% energy) and low protein diet to decrease excess intake of isoleucine, leucine, lysine, tryptophan, and valine (45). Patients even need to avoid MCT oil because $\beta$-oxidation of all fatty acid chain lengths is compromised (64). Additionally, patients require avoidance of fasting, adequate energy to prevent catabolism as well as supplementation with riboflavin and carnitine (43). Acute decompensation should be treated promptly with intravenous fluids and carnitine to restore anabolism. Ketone bodies represent a source of energy that patients with MADD can metabolize, and small trials have shown some success, especially relative to cardiac function (45). However, larger studies are necessary to confirm initial results.

\section{Therapies under investigation}

\section{Tribeptanoin}

Triheptanoin (Ultragenyx Pharmaceutical Inc., Novato, California, USA) is a triglyceride of three seven-carbon fatty acids that has been investigated for use as an alternative fuel source. Hydrolyzed in the small intestine to three molecules of heptanoic acid, the heptanoic acid then enters $\beta$-oxidation to produce acetyl-CoA and propionylCoA, which then can enter the citric acid cycle. In the liver, pentanoyl-CoA can serve as an anaplerotic substrate and generates 5 -carbon ketone bodies ( $\beta$-hydroxypentanoate and $\beta$-ketopentanoate), which can be utilized by peripheral tissues (65). Triheptanoin may also suppress lipolysis and accumulation of toxic metabolites in LCFAODs.

Reports of anaplerotic therapy with triheptanoin have included three VLCADD patients who had improvement in cardiac symptoms, muscle weakness and fatigue, hypoglycemia, and hepatomegaly within the first month of treatment. Rhabdomyolysis was not improved $(66,67)$. Subsequent studies showed similar effects in LCHADD, TFPD, CPT2D, and CPT1D, though LCHADD-associated retinopathy was not improved with treatment (67). A compassionate use protocol of 11 VLCADD, 5 LCHADD, 2 TFPD, 3 CPT2D, and 1 CACTD patients demonstrated triheptanoin to be well tolerated and reduced hospitalizations, myopathy, and hypoglycemia, with one patient having continued cardiomyopathy (68). Additional clinical studies including a double blind FDA Phase II are underway (68). Clinical long-term, randomized studies comparing outcomes 
between triheptanoin and standard therapy with MCT oil are ongoing.

\section{Gene transcription activation}

There have been a number of reports proposing the use of bezafibrate, which is currently used in treating hyperlipidemia to reduce LDL and triglyeride levels and increase HDL levels. Bezafibrate is a peroxisome proliferator-activated receptor (PPAR) agonist that promotes transcription of many genes including CPT2D and VLCADD. Clinical trials with CPT2D and VLCADD patients have shown varying effects ranging from a reduction in episodes of rhabdomyolysis and creatine kinase levels and with improvements in quality of life measures to patients who did not show improvement of clinical symptoms or fatty acid oxidation during exercise $(69,70)$. A recent open label study in VLCADD and CPT2D patients demonstrated decreased and increased myopathic attacks, increased and decreased creatine kinase levels, plasma acylcarnitine levels between patients, but did show consistent improvements in quality of life scores (71). More clinical studies are clearly needed in development of this potential treatment.

\section{Conclusions}

The FAOD are a group of autosomal recessive disorders associated with significant morbidity and mortality, but early diagnosis on NBS and early initiation of treatment are improving outcomes. The wide range of clinical presentations of these disorders ranges from lethal neonatal cardiomyopathy in the first days of life to chronic skeletal myopathy or even potentially benign traits. Current treatment may include nutrition management with a low-fat diet, MCT oil supplementation, aggressive treatment during illness, and potentially carnitine supplementation. Even with the significant advances made to date, there still remains significant risk for symptoms, especially rhabdomyolysis and cardiomyopathy. There is a need for clinical studies including randomized, controlled, therapeutic trials to continue to evaluate current understanding and to implement future therapies.

\section{Acknowledgements}

We would like to thank Jerry Vockley, MD PhD for providing critical feedback on the figure 'Overview of
Mitochondrial Fatty Acid Oxidation Metabolism'.

\section{Footnote}

Conflicts of Interest: The authors have no conflicts of interest to declare.

\section{References}

1. Rohr F, Calcar SV. Very Long Chain Acyl CoA Dehydrogenase Deficiency (VLCADD). Genetic Metabolic Dietitians International: Nutrition Guidelines. 2008. Available online: http://gmdi.org/Resources/ Nutrition-Guidelines/VLCAD. Accessed August 132018.

2. Miller MJ, Burrage LC, Gibson JB, et al. Recurrent ACADVL molecular findings in individuals with a positive newborn screen for very long chain acyl-coA dehydrogenase (VLCAD) deficiency in the United States. Mol Genet Metab 2015;116:139-45.

3. Collins SA, Sinclair G, McIntosh S, et al. Carnitine palmitoyltransferase 1A (CPT1A) P479L prevalence in live newborns in Yukon, Northwest Territories, and Nunavut. Mol Genet Metab 2010;101:200-4.

4. Clemente FJ, Cardona A, Inchley CE, et al. A Selective Sweep on a Deleterious Mutation in CPT1A in Arctic Populations. Am J Hum Genet 2014;95:584-9.

5. Prasad C, Johnson JP, Bonnefont JP, et al. Hepatic carnitine palmitoyl transferase 1 (CPT1 A) deficiency in North American Hutterites (Canadian and American): evidence for a founder effect and results of a pilot study on a DNA-based newborn screening program. Mol Genet Metab 2001;73:55-63.

6. Wieser T, Deschauer M, Olek K, et al. Carnitine palmitoyltransferase II deficiency: molecular and biochemical analysis of 32 patients. Neurology 2003;60:1351-3.

7. Gallant NM, Leydiker K, Tang H, et al. Biochemical, molecular, and clinical characteristics of children with short chain acyl-CoA dehydrogenase deficiency detected by newborn screening in California. Mol Genet Metab 2012;106:55-61.

8. van Maldegem BT, Duran M, Wanders RJ, et al. Clinical, biochemical, and genetic heterogeneity in short-chain acyl-coenzyme A dehydrogenase deficiency. Jama 2006;296:943-52.

9. Newborn screening: toward a uniform screening panel and system. Genet Med 2006;8 Suppl 1:1S-252S.

10. Service GD. NHS newborn blood spot (NBS) screening 
programme. Government Digital Service, United Kingdom. 2013. Available online: https://www.gov.uk/government/ collections/newborn-blood-spot-screening-programmesupporting-publications. Accessed August 162018.

11. Merritt JL 2nd, Vedal S, Abdenur JE, et al. Infants suspected to have very-long chain acyl-CoA dehydrogenase deficiency from newborn screening. Mol Genet Metab 2014;111:484-92.

12. Americal College of Medical Genetics Newborn Screening Expert Group. Newborn screening: toward a uniform screening panel and system--executive summary. Pediatrics 2006;117:S296-307.

13. Wilson CJ, Champion MP, Collins JE, et al. Outcome of medium chain acyl-CoA dehydrogenase deficiency after diagnosis. Arch Dis Child 1999;80:459-62.

14. Arnold GL, Saavedra-Matiz CA, Galvin-Parton PA, et al. Lack of genotype-phenotype correlations and outcome in MCAD deficiency diagnosed by newborn screening in New York State. Mol Genet Metab 2010;99:263-8.

15. Wilcken B, Haas M, Joy P, et al. Outcome of neonatal screening for medium-chain acyl-CoA dehydrogenase deficiency in Australia: a cohort study. Lancet 2007;369:37-42.

16. Matern D, Rinaldo P. Medium-Chain Acyl-Coenzyme A Dehydrogenase Deficiency. In: Adam MP, Ardinger HH, Pagon RA, et al. editors. GeneReviews((R)). Seattle (WA): University, of Washington, 1993.

17. Nennstiel-Ratzel U, Arenz S, Maier EM, et al. Reduced incidence of severe metabolic crisis or death in children with medium chain acyl-CoA dehydrogenase deficiency homozygous for c.985A $>\mathrm{G}$ identified by neonatal screening. Mol Genet Metab 2005;85:157-9.

18. Chace DH, Kalas TA, Naylor EW. The application of tandem mass spectrometry to neonatal screening for inherited disorders of intermediary metabolism. Annu Rev Genomics Hum Genet 2002;3:17-45.

19. Wilcken B, Wiley V, Hammond J, et al. Screening newborns for inborn errors of metabolism by tandem mass spectrometry. N Engl J Med 2003;348:2304-12.

20. Zytkovicz TH, Fitzgerald EF, Marsden D, et al. Tandem mass spectrometric analysis for amino, organic, and fatty acid disorders in newborn dried blood spots: a two-year summary from the New England Newborn Screening Program. Clin Chem 2001;47:1945-55.

21. Spiekerkoetter U, Tenenbaum T, Heusch A, et al. Cardiomyopathy and pericardial effusion in infancy point to a fatty acid b-oxidation defect after exclusion of an underlying infection. Pediatr Cardiol 2003;24:295-7.
22. Bonnet D, de Lonlay P, Gautier I, et al. Efficiency of metabolic screening in childhood cardiomyopathies. Eur Heart J 1998;19:790-3.

23. Bonnet D, Martin D, Pascale De L, et al. Arrhythmias and conduction defects as presenting symptoms of fatty acid oxidation disorders in children. Circulation 1999;100:2248-53.

24. Hoffman JD, Steiner RD, Paradise L, et al. Rhabdomyolysis in the military: recognizing late-onset very long-chain acyl Co-A dehydrogenase deficiency. Mil Med 2006;171:657-8.

25. Kluge S, Kuhnelt P, Block A, et al. A young woman with persistent hypoglycemia, rhabdomyolysis, and coma: recognizing fatty acid oxidation defects in adults. Crit Care Med 2003;31:1273-6.

26. Pena LD, van Calcar SC, Hansen J, et al. Outcomes and genotype-phenotype correlations in 52 individuals with VLCAD deficiency diagnosed by NBS and enrolled in the IBEM-IS database. Mol Genet Metab 2016;118:272-81.

27. Sperk A, Mueller M, Spiekerkoetter U. Outcome in six patients with mitochondrial trifunctional protein disorders identified by newborn screening. Mol Genet Metab 2010;101:205-7.

28. Spiekerkoetter U, Lindner M, Santer R, et al. Management and outcome in 75 individuals with long-chain fatty acid oxidation defects: results from a workshop. J Inherit Metab Dis 2009;32:488-97.

29. Sander J, Sander S, Steuerwald U, et al. Neonatal screening for defects of the mitochondrial trifunctional protein. Mol Genet Metab 2005;85:108-14.

30. Wilcken B. Fatty acid oxidation disorders: outcome and long-term prognosis. J Inherit Metab Dis 2010;33:501-6.

31. Bennett MJ, Santani AB. Carnitine Palmitoyltransferase $1 \mathrm{~A}$ Deficiency. In: Pagon RA, Adam MP, Ardinger HH, et al. editors. GeneReviews(R). Seattle (WA): University of Washington, 1993.

32. Bonnefont JP, Djouadi F, Prip-Buus C, et al. Carnitine palmitoyltransferases 1 and 2: biochemical, molecular and medical aspects. Mol Aspects Med 2004;25:495-520.

33. Gillingham MB, Hirschfeld M, Lowe S, et al. Impaired fasting tolerance among Alaska native children with a common carnitine palmitoyltransferase $1 \mathrm{~A}$ sequence variant. Mol Genet Metab 2011;104:261-4.

34. Yang BZ, Mallory JM, Roe DS, et al. Carnitine/ acylcarnitine translocase deficiency (neonatal phenotype): successful prenatal and postmortem diagnosis associated with a novel mutation in a single family. Mol Genet Metab 2001;73:64-70. 
35. Pierre G, Macdonald A, Gray G, et al. Prospective treatment in carnitine-acylcarnitine translocase deficiency. J Inherit Metab Dis 2007;30:815.

36. Al-Sannaa NA, Cheriyan GM. Carnitine-acylcarnitine translocase deficiency. Clinical course of three Saudi children with a severe phenotype. Saudi Med J 2010;31:931-4.

37. Spiekerkoetter U. Mitochondrial fatty acid oxidation disorders: clinical presentation of long-chain fatty acid oxidation defects before and after newborn screening. J Inherit Metab Dis 2010;33:527-32.

38. Rubio-Gozalbo ME, Bakker JA, Waterham HR, et al. Carnitine-acylcarnitine translocase deficiency, clinical, biochemical and genetic aspects. Mol Aspects Med 2004;25:521-32.

39. Lopriore E, Gemke RJ, Verhoeven NM, et al. Carnitineacylcarnitine translocase deficiency: phenotype, residual enzyme activity and outcome. Eur J Pediatr 2001;160:101-4.

40. Longo N, Amat di San Filippo C, Pasquali M. Disorders of carnitine transport and the carnitine cycle. Am J Med Genet C Semin Med Genet 2006;142C:77-85.

41. El-Hattab AW. Systemic Primary Carnitine Deficiency. In: Pagon RA, Adam MP, Ardinger HH, et al. editors. GeneReviews(R). Seattle (WA): University of Washington, 1993.

42. Pedersen CB, Kolvraa S, Kolvraa A, et al. The ACADS gene variation spectrum in 114 patients with short-chain acyl-CoA dehydrogenase (SCAD) deficiency is dominated by missense variations leading to protein misfolding at the cellular level. Hum Genet 2008;124:43-56.

43. Olsen RK, Olpin SE, Andresen BS, et al. ETFDH mutations as a major cause of riboflavin-responsive multiple acyl-CoA dehydrogenation deficiency. Brain 2007;130:2045-54.

44. Wilson GN, de Chadarevian JP, Kaplan P, et al. Glutaric aciduria type II: review of the phenotype and report of an unusual glomerulopathy. Am J Med Genet 1989;32:395-401.

45. Angle B, Burton BK. Risk of sudden death and acute lifethreatening events in patients with glutaric acidemia type II. Mol Genet Metab 2008;93:36-9.

46. Singla M, Guzman G, Griffin AJ, et al. Cardiomyopathy in multiple Acyl-CoA dehydrogenase deficiency: a clinicopathological correlation and review of literature. Pediatr Cardiol 2008;29:446-51.

47. Vredendaal PJ, van den Berg IE, Malingre HE, et al. Human short-chain L-3-hydroxyacyl-CoA dehydrogenase: cloning and characterization of the coding sequence. Biochem Biophys Res Commun 1996;223:718-23.
48. Vredendaal PJ, van den Berg IE, Stroobants AK, et al. Structural organization of the human short-chain L-3hydroxyacyl-CoA dehydrogenase gene. Mamm Genome 1998;9:763-8.

49. Clayton PT, Eaton S, Aynsley-Green A, et al. Hyperinsulinism in short-chain L-3-hydroxyacyl-CoA dehydrogenase deficiency reveals the importance of betaoxidation in insulin secretion. J Clin Invest 2001;108:457-65.

50. Flanagan SE, Patch AM, Locke JM, et al. Genome-wide homozygosity analysis reveals HADH mutations as a common cause of diazoxide-responsive hyperinsulinemichypoglycemia in consanguineous pedigrees. J Clin Endocrinol Metab 2011;96:E498-502.

51. Martins E, Cardoso ML, Rodrigues E, et al. Short-chain 3-hydroxyacyl-CoA dehydrogenase deficiency: the clinical relevance of an early diagnosis and report of four new cases. J Inherit Metab Dis 2011;34:835-42.

52. Kapoor RR, James C, Flanagan SE, et al. 3-Hydroxyacylcoenzyme A dehydrogenase deficiency and hyperinsulinemic hypoglycemia: characterization of a novel mutation and severe dietary protein sensitivity. J Clin Endocrinol Metab 2009;94:2221-5.

53. Li C, Chen P, Palladino A, et al. Mechanism of hyperinsulinism in short-chain 3-hydroxyacyl-CoA dehydrogenase deficiency involves activation of glutamate dehydrogenase. J Biol Chem 2010;285:31806-18.

54. Da'Torre SD, Creer MH, Pogwizd SM, et al. Amphipathic lipid metabolites and their relation to arrhythmogenesis in the ischemic heart. J Mol Cell Cardiol 1991;23 Suppl 1:11-22.

55. Frazier DM. Medium Chain Acyl CoA Dehydrogenase Deficiency (MCADD). Genetic Metabolic Dietitians International: Nutrition Guidelines. 2008. Available online: http://gmdi.org/Resources/Nutrition-Guidelines/ MCAD. Accessed August 132018.

56. Hove JV. Fatty Acid Oxidation Defects. In: Nutrition Management of Inherited Metabolic Diseases. Switzerland: Springer International Publishing, 2015.

57. Spiekerkoetter U, Lindner M, Santer R, et al. Treatment recommendations in long-chain fatty acid oxidation defects: consensus from a workshop. J Inherit Metab Dis 2009;32:498-505.

58. Rohr F. Nutrition Management of Fatty Acid Oxidation Disorders. Nutrition Management of Inherited Metabolic Diseases. Switzerland: Springer International Publishing, 2015.

59. Pervaiz MA, Kendal F, Hegde M, et al. MCT oil-based diet reverses hypertrophic cardiomyopathy in a patient with very long chain acyl-coA dehydrogenase deficiency. 
Indian J Hum Genet 2011;17:29-32.

60. Sharef SW, Al-Senaidi K, Joshi SN. Successful Treatment of Cardiomyopathy due to Very Long-Chain Acyl-CoA Dehydrogenase Deficiency: First Case Report from Oman with Literature Review. Oman Med J 2013;28:354-6.

61. Agriculture USDo. National Nutrient Database for Standard Reference Legacy Release. USDA Food Composition Databases. Available online: http://www.nal. usda.gov/fnic/foodcomp/search/. Accessed August 132018.

62. Gillingham MB. Nutrition Studies in Long-Chain Fatty Acid Oxidation Disorders: Diet Composition and Monitoring. Nutrition Management of Inherited Metabolic Diseases. Switzerland: Springer International Publishing, 2015.

63. Gillingham MB, Weleber RG, Neuringer M, et al. Effect of optimal dietary therapy upon visual function in children with long-chain 3-hydroxyacyl CoA dehydrogenase and trifunctional protein deficiency. Mol Genet Metab 2005;86:124-33.

64. El-Gharbawy A, Vockley J. Inborn Errors of Metabolism with Myopathy: Defects of Fatty Acid Oxidation and the Carnitine Shuttle System. Pediatr Clin North Am 2018;65:317-35.

65. Deng S, Zhang GF, Kasumov T, et al. Interrelations

Cite this article as: Merritt JL 2nd, Norris M, Kanungo S. Fatty acid oxidation disorders. Ann Transl Med 2018;6(24):473. doi: 10.21037/atm.2018.10.57 between C4 ketogenesis, C5 ketogenesis, and anaplerosis in the perfused rat liver. J Biol Chem 2009;284:27799-807.

66. Roe CR, Sweetman L, Roe DS, et al. Treatment of cardiomyopathy and rhabdomyolysis in long-chain fat oxidation disorders using an anaplerotic odd-chain triglyceride. J Clin Invest 2002;110:259-69.

67. Roe CR, Mochel F. Anaplerotic diet therapy in inherited metabolic disease: therapeutic potential. J Inherit Metab Dis 2006;29:332-40.

68. Barone AR, DeWard SJ, Payne N, et al. Triheptanoin therapy for inherited disorders of fatty acid oxidation. Program for Society for Inherited Medical Disorders Annual Meeting. Abstracts. Mol Genet Metab 2012;105:304.

69. Bonnefont JP, Bastin J, Behin A, et al. Bezafibrate for an inborn mitochondrial beta-oxidation defect. N Engl J Med 2009;360:838-40.

70. Ørngreen MC, Madsen KL, Preisler N, et al. Bezafibrate in skeletal muscle fatty acid oxidation disorders: a randomized clinical trial. Neurology 2014;82:607-13.

71. Yamada K, Shiraishi H, Oki E, et al. Open-label clinical trial of bezafibrate treatment in patients with fatty acid oxidation disorders in Japan. Mol Genet Metab Rep 2018;15:55-63. 